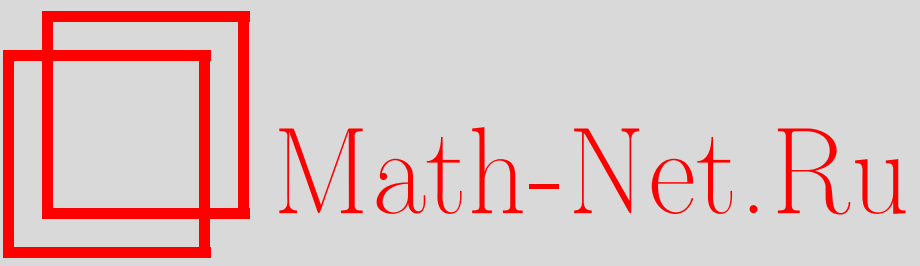

К. Роджерс, В. К. Шиф, Кинематика плоского движения идеальной жидкости, армированной волокном. Интегрируемая редукция и преобразование Беклунда, TMФ, 2003, том 137, номер 2, 281-292

DOI: https://doi.org/10.4213/tmf272

Использование Общероссийского математического портала Math-Net.Ru подразумевает, что вы прочитали и согласны с пользовательским соглашением

http://www.mathnet.ru/rus/agreement

Параметры загрузки:

IP: 52.6 .47 .48

26 апреля 2023 г., 10:34:01 


\section{КИНЕМАТИКА ПЛОСКОГО ДВИЖЕНИЯ ИДЕАЛЬНОЙ ЖИДКОСТИ, АРМИРОВАННОЙ ВОЛОКНОМ. ИНТЕГРИРУЕМАЯ РЕДУКЦИЯ И ПРЕОБРАЗОВАНИЕ БЕКЛУНДА}

Установлено, что кинематические ограничения на стационарное плоское движение идеальной жидкости, армированной волокном, можно объединить в одно нелинейное уравнение третьего порядка. Замечательно, что это уравнение допускает солитонную редукцию, связанную с классическим уравнением синус-Гордон. В этом случае кинематические условия обладают новым свойством дуальности и допускают преобразование Беклунда.

Ключевые слова: кинематика, армированные волокном материалы, преобразование Беклунда.

\section{1. ВВЕДЕНИЕ}

Армированные волокном материалы со смоляной матрицей имеют многочисленные технические применения, особенно при создании твердых легких слоистых структур со сложной геометрией оболочек. Математическая теория деформации армированных волокном материалов была сформулирована в основополагающей монографии Спенсера [1]. Особый интерес для промышленности представляет процесс формирования волоконно-смоляных систем, в котором смоляная матрица ведет себя подобно вязкой жидкости. В этой связи Халл с сотрудниками [2] предложили использовать для описания состояния волоконно-смоляных систем, находящихся в важной стадии формирования, модель идеальной жидкости, армированной волокном. Согласно этой модели весь объем жидкости заполнен линиями нерастяжимости вдоль направлений волокон. Наличие этих выделенных ориентаций внутри жидкости накладывает сильные кинематические ограничения на ее возможные движения.

Исследование кинематики идеальной жидкости, армированной волокном, было выполнено Спенсером [3]. При этом особое внимание было уделено плоским движениям, выделенным в том отношении, что они, по сушеству, определяются кинематическими

${ }^{*}$ School of Mathematics, The University of New South Wales, Sydney, NSW 2052, Australia. E-mail: colinr@maths.unsw.edu.au; schief@maths.unsw.edu.au 
условиями. В настояшей работе, используя геометрические методы, мы покажем, что в стационарном случае эти условия можно представить в форме одного нелинейного уравнения третьего порядка. Замечательно, что это уравнение допускает солитонную редукцию, ассоциирующуюся с автопреобразованием Беклунда для знаменитого уравнения синус-Гордон. Показано, что в этом случае кинематические условия имеют новое свойство дуальности. Строится преобразование Беклунда, позволяюшее генерировать геометрии волокон и поля скоростей, связанные с многосолитонными решениями кинематических уравнений и решениями бриферного типа.

\section{2. КИНЕМАТИЧЕСКИЕ УСЛОВИЯ}

Предложенная в [1], [3] идеальная модель представляет собой модель несжимаемой жидкости, нерастяжимой вдоль линий “волокон”, заполняющих весь объем жидкости, которая их конвективно переносит. Здесь мы обращаемся к условиям нерастяжимости и конвекции с самого начала. Итак, пусть $\mathbf{r}=\mathbf{r}\left(\mathbf{r}_{0}, t\right)$ обозначает радиус-вектор частицы жидкости в момент времени $t, \mathbf{r}_{0}$ - ее радиус-вектор в начальный момент времени, т.е. $\mathbf{r}\left(\mathbf{r}_{0}, 0\right)=\mathbf{r}_{0}$. Скорость частицы есть $\mathbf{q}=d \mathbf{r} / d t=\dot{\mathbf{r}}$. Обозначим через $\mathbf{t}=\mathbf{t}(\mathbf{r}, t)$ зависящий от времени единичный касательный к волокнам вектор. Рассмотрим при $t=0$ интегральную кривую $\Gamma(0): \mathbf{r}_{0}=\mathbf{r}_{0}(\sigma)$ векторного поля $\mathbf{t}$, определяемую соотношениem

$$
\frac{\partial \mathbf{r}_{0}}{\partial \sigma} \| \mathbf{t}_{0}, \quad \mathbf{t}_{0}=\mathbf{t}\left(\mathbf{r}_{0}, 0\right),
$$

параметр $\sigma$ маркирует частицы на кривой $\Gamma(0)$. Требуется, чтобы в процессе эволюции кривой, т.е. при движении частиц, первоначально находившихся на кривой $Г(0)$, кривая $\Gamma(t)$, образованная этими частицами, оставалась интегральной кривой, т.е.

$$
\frac{\partial \mathbf{r}}{\partial \sigma}=\lambda \mathbf{t}=\lambda \frac{\delta \mathbf{r}}{\delta s}
$$

где $\delta / \delta s=\mathbf{t} \cdot \nabla$ - производная по направлению вдоль кривой $Г$. Длина дуги $s$ этой кривой связана с величиной $\lambda$ соотношением

$$
s(\sigma, t)=\int_{\sigma_{0}}^{\sigma} \lambda d \tilde{\sigma}+s\left(\sigma_{0}, t\right) .
$$

Законы эволюции во времени для $\lambda$ и $\mathbf{t}$ получаются следуюшим образом. С одной стороны,

$$
\frac{d}{d t} \lambda^{2}=\frac{d}{d t}\left(\frac{\partial \mathbf{r}}{\partial \sigma}\right)^{2}
$$

откуда

$$
\lambda \dot{\lambda}=\frac{\partial \mathbf{r}}{\partial \sigma} \cdot \frac{\partial \dot{\mathbf{r}}}{\partial \sigma}=\frac{\partial \mathbf{r}}{\partial \sigma} \cdot \frac{\partial \mathbf{q}}{\partial \sigma}=\lambda^{2} \frac{\delta \mathbf{r}}{\delta s} \cdot \frac{\delta \mathbf{q}}{\delta s},
$$

так что

$$
\dot{\lambda}=\lambda \mathbf{t} \cdot \frac{\delta \mathbf{q}}{\delta s}
$$


Отсюда следует, что необходимым и достаточным условием нерастяжимости интегральной кривой $\Gamma$ (волокна) является условие $\dot{\lambda}=0$, т.е. условие

$$
\mathbf{t} \cdot \frac{\delta \mathbf{q}}{\delta s}=0
$$

С другой стороны, в силу $(2.2)$

$$
\frac{d}{d t}\left(\frac{\partial \mathbf{r}}{\partial \sigma}\right)=\dot{\lambda} \mathbf{t}+\lambda \dot{\mathbf{t}},
$$

откуда, с учетом требования нерастяжимости $\dot{\lambda}=0$, следует

$$
\frac{\partial \mathbf{q}}{\partial \sigma}=\lambda \dot{\mathbf{t}},
$$

или

$$
\dot{\mathbf{t}}=\frac{\delta \mathbf{q}}{\delta s} .
$$

Последнее уравнение дает конвективное кинематическое условие, установленное в работе [3], а именно

$$
\frac{\partial \mathbf{t}}{\partial t}+(\mathbf{q} \cdot \nabla) \mathbf{t}=(\mathbf{t} \cdot \nabla) \mathbf{q} .
$$

Заметим, что это кинематическое условие тем более влечет за собой условие нерастяжимости (2.4). Этот факт был отмечен Спенсером [1].

Кинематическое условие (2.5) должно быть добавлено к обычному уравнению непрерывности

$$
\operatorname{div} \mathbf{q}=0 .
$$

В данной работе нас будет интересовать именно эта система кинематических условий $(2.5),(2.6)$. При этом мы ограничимся кинематикой стационарного плоского движения. Отдельные стороны пространственных движений исследовались в работе [4].

\section{3. ГЕОМЕТРИЯ СТАЦИОНАРНОГО ПЛОСКОГО ДВИЖЕНИЯ}

Согласно Спенсеру [3] двумерные потоки идеальной жидкости, армированной волокном, по существу, определяются кинематическими соображениями. Именно, давление $p$ и натяжение $T$ в направлении волокон всегда можно определить таким образом, чтобы уравнения движения удовлетворялись. Соответственно плоское движение занимает выделенное положение и исследование его кинематики приобретает особую важность. В этом разделе мы покажем, что кинематические условия для идеальной жидкости, армированной волокном, можно сформулировать в виде одного нелинейного уравнения третьего порядка. Затем в разделе 4 будет показано, что это уравнение замечательным образом содержит вариант хорошо известной интегрируемой системы в теории солитонов. 
Перейдем к изучению плоской кинематики. Ниже $\mathbf{t}$ и $\mathbf{n}$ обозначают соответственно единичный касательный вектор и вектор главной нормали к волокну обшего положения. Уравнения Серре-Френе для этого репера имеют вид

$$
\begin{aligned}
& \frac{\delta}{\delta s}\left(\begin{array}{l}
\mathbf{t} \\
\mathbf{n}
\end{array}\right)=\left(\begin{array}{cc}
0 & \kappa \\
-\kappa & 0
\end{array}\right)\left(\begin{array}{l}
\mathbf{t} \\
\mathbf{n}
\end{array}\right), \\
& \frac{\delta}{\delta n}\left(\begin{array}{l}
\mathbf{t} \\
\mathbf{n}
\end{array}\right)=\left(\begin{array}{cc}
0 & \theta \\
-\theta & 0
\end{array}\right)\left(\begin{array}{l}
\mathbf{t} \\
\mathbf{n}
\end{array}\right),
\end{aligned}
$$

где

$$
\frac{\delta}{\delta s}=\mathbf{t} \cdot \nabla, \quad \frac{\delta}{\delta n}=\mathbf{n} \cdot \nabla
$$

обозначают производные соответственно по касательному направлению и по направлению главной нормали, так что градиент $\nabla$ можно представить в виде

$$
\nabla=\mathbf{t} \frac{\delta}{\delta s}+\mathbf{n} \frac{\delta}{\delta n} .
$$

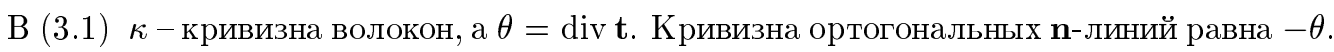
Применяя коммутационное соотношение

$$
\frac{\delta^{2}}{\delta n \delta s}-\frac{\delta^{2}}{\delta s \delta n}=\kappa \frac{\delta}{\delta s}+\theta \frac{\delta}{\delta n}
$$

к системе (3.1), получаем

$$
\frac{\delta \kappa}{\delta n}-\frac{\delta \theta}{\delta s}=\kappa^{2}+\theta^{2}
$$

Общее решение последнего геометрического условия может быть параметризовано с использованием

$$
\kappa=\frac{\delta \varphi}{\delta s}, \quad \theta=\frac{\delta \varphi}{\delta n},
$$

где $\varphi$ - неизвестная пока функция. Однако геометрический смысл $\varphi$ как угла становится немедленно очевидным, если проинтегрировать систему уравнений Серре-Френе (3.1) в терминах декартовых координат $(x, y)$ и соответствуюших единичных касательных векторов $\mathbf{e}_{(x)}$ и $\mathbf{e}_{(y)}:$

$$
\begin{aligned}
\mathbf{t} & =\mathbf{e}_{(x)} \cos \varphi+\mathbf{e}_{(y)} \sin \varphi \\
\mathbf{n} & =-\mathbf{e}_{(x)} \sin \varphi+\mathbf{e}_{(y)} \cos \varphi
\end{aligned}
$$

Если представить теперь скорость жидкости q в виде разложения

$$
\mathbf{q}=v \mathbf{t}+w \mathbf{n}
$$

и затем подставить его в кинематические условия (2.5) и (2.6), то для стационарного движения получим

$$
\frac{\delta v}{\delta s}=\kappa w, \quad \frac{\delta w}{\delta s}=\theta w, \quad \frac{\delta w}{\delta n}=-\theta v
$$

где было использовано соотношение $\kappa+\operatorname{div} \mathbf{n}=0$, справедливое для плоской геометрии. 
Естественно использовать линии волокон и ортогональные к ним траектории в качестве координатных линий ортогональной системы координат на плоскости. В этом случае, если переменные $s$ и $n$ параметризуют соответственно $\mathbf{t}-$ и $\mathbf{n}$-линии, метрика плоскости принимает вид

$$
\mathrm{I}=\phi^{2} d s^{2}+\psi^{2} d n^{2}
$$

где частные производные и производные по направлениям связаны соотношениями

$$
\frac{\partial}{\partial s}=\phi \frac{\delta}{\delta s}, \quad \frac{\partial}{\partial n}=\psi \frac{\delta}{\delta n} .
$$

Таким образом, если $\mathbf{r}$ является точкой общего положения на плоскости, то

$$
\frac{\delta \mathbf{r}}{\delta s}=\mathbf{t}=\frac{1}{\phi} \frac{\partial \mathbf{r}}{\partial s}, \quad \frac{\delta \mathbf{r}}{\delta n}=\mathbf{n}=\frac{1}{\psi} \frac{\partial \mathbf{r}}{\partial n} .
$$

Согласно (3.4) геометрические величины $\kappa$ и $\theta$ теперь можно представить следующим образом:

$$
\kappa=\frac{1}{\phi} \frac{\partial \varphi}{\partial s}, \quad \theta=\frac{1}{\psi} \frac{\partial \varphi}{\partial n} .
$$

Подставляя (3.7) в коммутационное соотношение (3.2), получаем

$$
\frac{1}{\psi} \frac{\partial}{\partial n}\left(\frac{1}{\phi} \frac{\partial}{\partial s}\right)-\frac{1}{\phi} \frac{\partial}{\partial s}\left(\frac{1}{\psi} \frac{\partial}{\partial n}\right)=\frac{\kappa}{\phi} \frac{\partial}{\partial s}+\frac{\theta}{\psi} \frac{\partial}{\partial n},
$$

откуда, используя (3.8), находим

$$
\psi_{s}=\varphi_{n} \phi, \quad \phi_{n}=-\varphi_{s} \psi
$$

Здесь и в дальнейшем нижние индексы обозначают частные производные.

Удобно ввести величину $\rho$, определяемую соотношениями

$$
\frac{\delta \rho}{\delta s}=1, \quad \frac{\delta \rho}{\delta n}=-\frac{v}{w} .
$$

Использование соотношения коммутации (3.2) и первого и второго кинематических условий из (3.5) показывает, что соотношения (3.10) являются совместными. Заметим, что первое кинематическое условие из (3.5) есть в точности условие нерастяжимости (2.4). В силу первого равенства из (3.10) величина $\rho$ представляет собой длину дуги вдоль волокон. Более того, комбинируя соотношения (3.10), получаем

$$
\mathbf{q} \cdot \nabla \rho=0
$$

тогда как из совместности второго и третьего кинематических условий из (3.5) с учетом (3.2) вытекает, что

$$
\mathbf{q} \cdot \nabla \theta=0,
$$

откуда

$$
\theta=\operatorname{div} \mathbf{t}=R(\rho) .
$$


Как теперь видно, следует положить

$$
R(\rho)=\frac{\Psi^{\prime}(\rho)}{\Psi(\rho)},
$$

и тогда из второго соотношения (3.8) и первого равенства (3.9) следует, что

$$
\frac{\psi_{s}}{\psi}=\frac{\Psi^{\prime}(\rho)}{\Psi(\rho)} \phi
$$

в то время как из первого соотношения (3.10) следует, что $\phi=\rho_{s}$, откуда

$$
\psi=\Psi(\rho)
$$

При этом в последнем соотношении мы опустили произвольную функцию интегрирования $N(n)$; это можно сделать без потери общности, поскольку эту функцию можно устранить подходящей репараметризацией n-линий. С учетом (3.11) и (3.12) второе соотношение в (3.8) сводится к

$$
\varphi_{n}=\Psi^{\prime}(\rho),
$$

тогда как оставшееся второе условие (3.9) принимает вид

$$
\varphi_{s}=-\frac{\rho_{s n}}{\Psi(\rho)}
$$

Исключая $\varphi$ из двух последних соотношений, получаем следуюший результат.

ТЕОРемА 1. Кинематика стационарного плоского движения идеальной жкидкости, армированной волокном, подчиняется нелинейному уравнению третьего порядка для длины дуги $\rho$ вдоль волокон:

$$
\left(\frac{\rho_{s n}}{\Psi(\rho)}\right)_{n}+\Psi^{\prime \prime}(\rho) \rho_{s}=0
$$

где $\Psi$ - произвольная функиия $\rho$. Однопараметрическое семейство волокон $\mathbf{r}(s, n=$ const) получается интегрированием пары совместных уравнений

$$
\mathbf{r}_{s}=\rho_{s} \mathbf{t}, \quad \mathbf{r}_{n}=\Psi(\rho) \mathbf{n},
$$

тогда как скорость жиидкости дается формулой

$$
\mathbf{q}=-\rho_{n} \mathbf{t}+\Psi(\rho) \mathbf{n}
$$

Решение совместных уравнений

$$
\varphi_{s}=-\frac{\rho_{s n}}{\Psi(\rho)}, \quad \varphi_{n}=\Psi^{\prime}(\rho)
$$

приводит к следующему представлению единичного касательного вектора $\mathbf{t}$ и вектора главной нормали $\mathbf{n} к$ волокнам:

$$
\mathbf{t}=\left(\begin{array}{c}
\cos \varphi \\
\sin \varphi
\end{array}\right), \quad \mathbf{n}=\left(\begin{array}{c}
-\sin \varphi \\
\cos \varphi
\end{array}\right)
$$




\section{4. ИНТЕГРИРУЕМАЯ РЕДУКЦИЯ. ГЕНЕРАЦИЯ РАСПРЕДЕЛЕНИЙ ВОЛОКОН}

Для получения связи с теорией солитонов оказывается удобным переформулировать уравнение третьего порядка (3.13) в виде системы двух уравнений

$$
\rho_{s n}=\mu \Psi(\rho), \quad \mu_{n}=-\Psi^{\prime \prime}(\rho) \rho_{s} .
$$

Последнее уравнение допускает первый интеграл

$$
\mu^{2}-\alpha \rho_{s}^{2}=I(s)
$$

если функция $\Psi$ удовлетворяет условию

$$
\Psi^{\prime \prime}(\rho)+\alpha \Psi(\rho)=0,
$$

где $\alpha$-произвольная постоянная. Используя подходящую репараметризацию линий волокон, функцию $I$ можно привести к виду, при котором она принимает значения $0, \pm 1$. В частности, если $\alpha=I=1$ и

$$
\Psi(\rho)=\sin \rho,
$$

представление

$$
\mu=\epsilon \operatorname{ch} \omega, \quad \rho_{s}=\epsilon \operatorname{sh} \omega, \quad \epsilon^{2}=1
$$

соотношения (4.2) приводит к следуюшему следствию из теоремы 1.

СлЕДСтвиЕ. Въбором $\Psi(\rho)=\sin \rho$ уравнение (3.13) сводится $к$ системе уравнений

$$
\rho_{s}=\epsilon \operatorname{sh} \omega, \quad \omega_{n}=\sin \rho,
$$

при этом скорость жидкости дается формулой

$$
\mathbf{q}=-\mathbf{t} \rho_{n}+\mathbf{n} \sin \rho
$$

где пара векторов $(\mathbf{t}, \mathbf{n})$ определяется формулами (3.14), причем

$$
\varphi_{s}=-\epsilon \operatorname{ch} \omega, \quad \varphi_{n}=\cos \rho .
$$

Метрика плоскости может бъть приведена к виду

$$
\mathrm{I}=\rho_{s}^{2} d s^{2}+\omega_{n}^{2} d n^{2},
$$

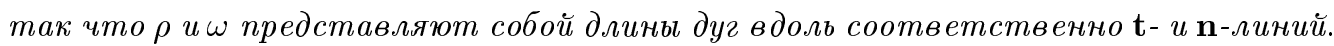

Если слагаемое $\epsilon \operatorname{sh} \omega$ в (4.4) заменить на $\sin \omega$, то получаются классические уравнения Беклунда для поверхностей постоянной отрицательной гауссовой кривизны [5]. Уравнение Гаусса [6] для таких псевдосферических поверхностей - это интегрируемое уравнение синус-Гордон. В данном контексте легко проверить, что $\rho+i \omega$ подчиняются уравнению синус-Гордон

$$
(\rho+i \omega)_{s n}=\epsilon \sin (\rho+i \omega)
$$


Система уравнений (4.4) обеспечивает совместность пары

$$
\begin{aligned}
\Phi_{s} & =\frac{\epsilon}{2}\left(\begin{array}{cc}
\operatorname{tg} \nu \operatorname{sh} \omega & -\frac{\operatorname{ch} \omega}{\cos \nu} \\
\frac{\operatorname{ch} \omega}{\cos \nu} & -\operatorname{tg} \nu \operatorname{sh} \omega
\end{array}\right) \Phi, \\
0 & \cos (\rho-\nu) \\
\Phi_{n} & =\frac{1}{2}\left(\begin{array}{cc}
0 & 0
\end{array}\right) \Phi
\end{aligned}
$$

при любом значении постоянного параметра $\nu$. Фактически эта пара является “линейным представлением" нелинейной системы (4.4) в смысле теории солитонов и допускает соответствуюшее преобразование Дарбу [5]. Заметим в этой связи, что при $\nu=0$ фундаментальное решение уравнений (4.7) имеет вид

$$
\left.\Phi(\nu)\right|_{\nu=0}=\left(\begin{array}{cc}
\cos \frac{\varphi}{2} & \sin \frac{\varphi}{2} \\
-\sin \frac{\varphi}{2} & \cos \frac{\varphi}{2}
\end{array}\right)
$$

Таким образом, “волновая функция” Ф задает представление векторов $\mathbf{t}$ и $\mathbf{n}$ в терминах угла $\varphi$. Индуцированное преобразование Беклунда для распределения волокон на плоскости можно получить стандартным образом, используя формулу Сима-Тафеля [5], [7]. В данном контексте согласно этой формуле соотношение для радиус-вектора волокон содержится в выражении

$$
\left.\Phi^{-1} \frac{\partial \Phi}{\partial \nu}\right|_{\nu=0}
$$

Суммируем вышеприведенные результаты:

ТЕОрема 2. Любое решение $(\rho, \omega)$ интегрируемой системы (4.4) и соответствующие радиус-вектор волокон $\mathbf{r}$ и векторы $\mathbf{t}, \mathbf{n}$ можно отобразить в другое решение $\left(\rho^{\prime}, \omega^{\prime}\right)$ и соответствующее распределение волокон $\left(\mathbf{r}^{\prime}, \mathbf{t}^{\prime}, \mathbf{n}^{\prime}\right)$ преобразованием Беклунда

$$
\begin{gathered}
\rho^{\prime}=\rho+2 \operatorname{arctg}\left(\operatorname{ctg} \nu \frac{1+\xi^{2}}{1-\xi^{2}}\right), \\
\omega^{\prime}=-\omega+2 \operatorname{arcth}\left(\frac{2 \xi \sin \nu}{1+\xi^{2}}\right), \\
\varphi^{\prime}=\varphi+\chi, \quad \chi=-2 \operatorname{arctg}\left(\frac{2 \xi \cos \nu}{1-\xi^{2}}\right), \\
\mathbf{r}^{\prime}=\mathbf{r}+2 \operatorname{ctg} \nu \frac{1+\xi^{2}}{1-\xi^{2}} \cos \frac{\chi}{2}\left(\mathbf{t} \cos \frac{\chi}{2}+\mathbf{n} \sin \frac{\chi}{2}\right), \\
\mathbf{t}^{\prime}=\mathbf{t} \cos \chi+\mathbf{n} \sin \chi, \quad \mathbf{n}^{\prime}=-\mathbf{t} \sin \chi+\mathbf{n} \cos \chi
\end{gathered}
$$

где $\xi=\Phi^{1} / \Phi^{2}$ и $\epsilon^{\prime}=-\epsilon$. Здесь $\Phi^{i}$ обозначает компоненты векторнозначного решения $\Phi$ линейной системь уравнений (4.7).

Одним из важных проявлений этой симметрии между исходным распределением и его преобразованием Беклунда являются следующие тождества:

$$
\mathbf{r}^{\prime}-\mathbf{r}=\operatorname{tg}\left(\frac{\rho^{\prime}-\rho}{2}\right)\left(\mathbf{t}^{\prime}+\mathbf{t}\right)=\operatorname{cth}\left(\frac{\omega^{\prime}+\omega}{2}\right)\left(\mathbf{n}^{\prime}-\mathbf{n}\right)
$$




$$
\operatorname{tg} \frac{\chi}{2}+\operatorname{tg}\left(\frac{\rho^{\prime}-\rho}{2}\right) \operatorname{th}\left(\frac{\omega^{\prime}+\omega}{2}\right)=0
$$

Эти соотношения и вид метрики (4.6) дают также возможность предполагать, что волокна и ортогональные им траектории равноправны. И это действительно так. Именно, если ввести векторное поле

$$
\mathbf{w}=\mathbf{t} \operatorname{sh} \omega-\mathbf{n} \epsilon \omega_{s}
$$

то легко показать, что

$$
(\mathbf{w} \cdot \nabla) \mathbf{n}=(\mathbf{n} \cdot \nabla) \mathbf{w}, \quad \operatorname{div} \mathbf{w}=0 .
$$

Соответственно $\mathbf{n}$-линии можно рассматривать как нерастяжимые волокна, которые конвективно переносятся потоком w. Нерастяжимость n-линий подтверждает соотношение

$$
(\mathbf{w} \cdot \nabla) \omega=0
$$

Таким образом, нами установлена дуальность вида

$$
(\mathbf{t}, \mathbf{v}) \leftrightarrow(\mathbf{n}, \mathbf{w})
$$

Более того, можно показать, что такое свойство дуальности сушествует тогда и только тогда, когда функция $\Psi(\rho)$ удовлетворяет условию (4.3). Таким образом, интегрируемый случай выделен свойством дальности. При определенных обстоятельствах оба семейства волокон конвективно переносятся потоком жидкости. Этот случай естественным образом появляется в следующем разделе.

\section{5. СПЕЦИАЛЬНЫЕ ДВИЖЕНИЯ}

В качестве иллюстрации установленного ранее преобразования Беклунда выберем простейшее решение систем уравнений (4.4) и (4.5), а именно решение

$$
\rho=0, \quad \omega=0, \quad \varphi=n-s, \quad \epsilon=1,
$$

отвечающее вырожденному распределению волокон

$$
\mathbf{r}=0, \quad \mathbf{t}=\left(\begin{array}{c}
\cos (n-s) \\
\sin (n-s)
\end{array}\right), \quad \mathbf{n}=\left(\begin{array}{c}
-\sin (n-s) \\
\cos (n-s)
\end{array}\right)
$$

Для соответствуюшего решения линейной системы (4.7) имеем

$$
\xi=\operatorname{tg} \frac{\Xi}{2}, \quad \Xi=n \cos \nu-\frac{s}{\cos \nu},
$$

так что преобразования Беклунда для $\rho$ и $\omega$ имеют вид

$$
\rho^{\prime}=2 \operatorname{arctg}\left(\frac{\cos \nu}{\cos \Xi}\right), \quad \omega^{\prime}=2 \operatorname{arcth}(\sin \nu \sin \Xi)
$$

5 Теоретическая и математическая физика, т. 137, № 2, 2003 г. 

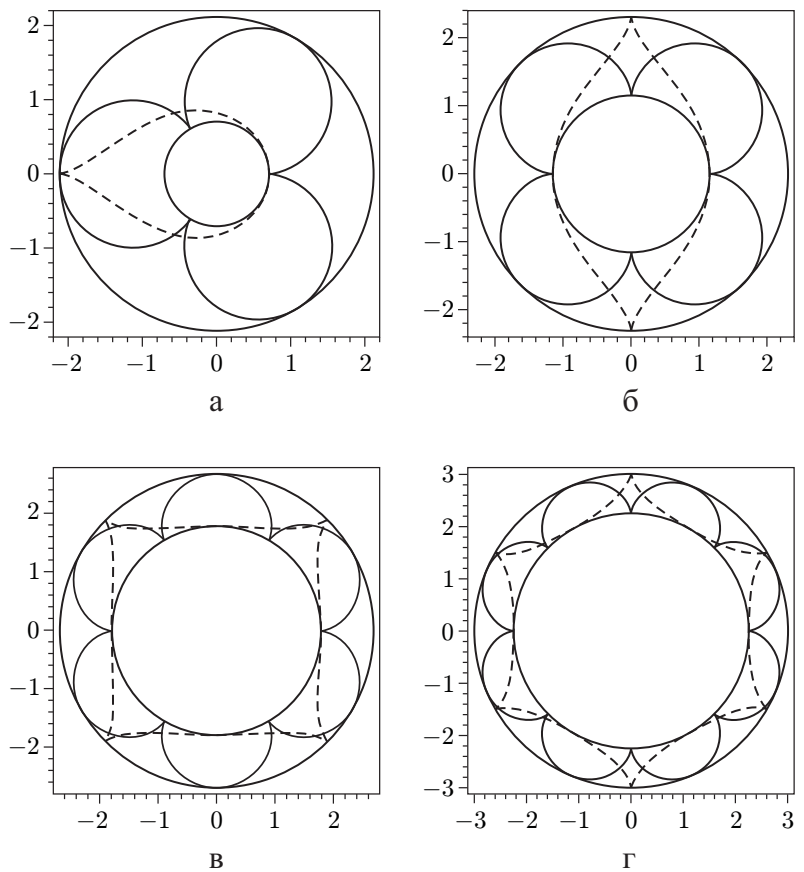

Рис. 1. Волокна (сплошные линии) и ортогональные им траектории (штриховые линии) при $\cos \nu=1 / 3(\mathrm{a}), 1 / 2$ (б), 2/3 (в), 3/4 (г).

и

$$
\chi=-2 \operatorname{arctg}(\cos \nu \operatorname{tg} \Xi) .
$$

Вычисленный по формуле из (4.9) радиус-вектор $\mathbf{r}^{\prime}$ имеет вид

$$
\mathbf{r}^{\prime}=\frac{2 \operatorname{ctg} \nu}{1-\sin ^{2} \nu \sin ^{2} \Xi}(\mathbf{t} \cos \Xi-\mathbf{n} \cos \nu \sin \Xi) .
$$

Соотношение

$$
\mathbf{r}^{\prime 2}=\frac{4 \operatorname{ctg}^{2} \nu}{1-\sin ^{2} \nu \sin ^{2} \Xi}
$$

показывает, что волокна заключены в кольцевой области, имеющей внутренний радиус $2|\operatorname{ctg} \nu|$ и внешний радиус $2 /|\sin \nu|$. Волокна в этой области одинаковы и получаются друг из друга врашениями вокруг начала координат. Если $\cos \nu$ является иррациональным числом, то одиночное волокно покрывает всю область, тогда как рациональным значениям $\cos \nu$ отвечают замкнутые волокна. Более того, можно показать, что

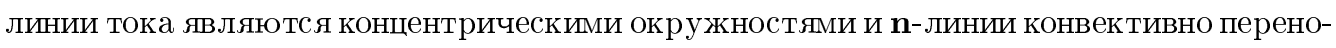
сятся тем же круговым потоком жидкости. Эти два утверждения являются “побочными продуктами" представленного ниже заключительного общего анализа. Различные замкнутые волокна, ортогональные им траектории и круговые линии тока изображены на рис.1. Видно, что линии волокон имеют точки поворота на внутренней границе. Кинематически допустимые деформации, при которых производные линий волокон терпят разрывы в некоторых точках, обсуждались в [1], [8]. 
Другая геометрия волокон получается при подстановке

$$
\nu \rightarrow i \nu+\frac{\pi}{2}, \quad\left(\mathbf{r}^{\prime}, \rho^{\prime}, \omega^{\prime}\right) \rightarrow i\left(\mathbf{r}^{\prime}, \rho^{\prime}, \omega^{\prime}\right)
$$

В этом случае $\rho^{\prime}$ и $\omega^{\prime}$ можно рассматривать как решения типа “бегушей волны", если $n$ отождествить со "временем". Действительно, $\rho^{\prime}$ описывает объект типа солитона, тогда как $\omega^{\prime}$ представляет собой кинк (см. рис. 2).
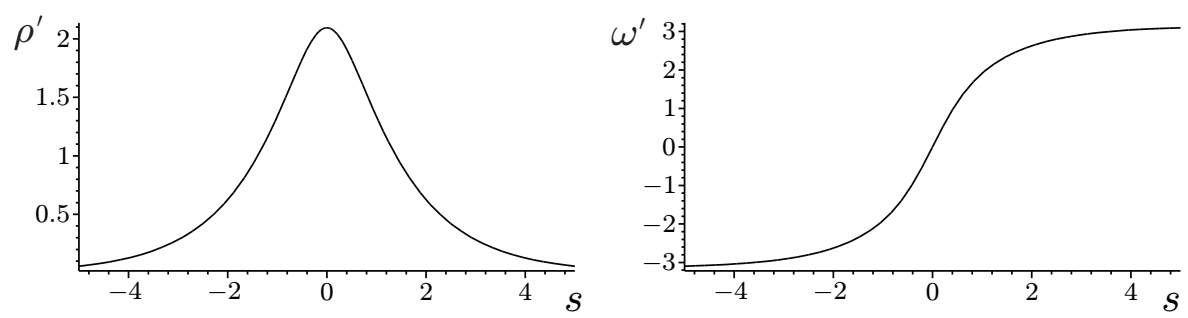

Рис. 2. Солитонное и кинковое решения интегрируемой системы (4.4).

Полученные выше решения нелинейной системы (4.4) зависят только от одной величины вида $z=n+c s$, где $c$ - произвольная константа. Поэтому естественно исследовать соответствуюшую редукцию симметрии в общем случае. Пусть $\rho$ - функция только $z$, тогда уравнение третьего порядка (3.13) сводится к уравнению

$$
\ddot{\rho}+\Psi(\rho) \Psi^{\prime}(\rho)=\tilde{c} \Psi(\rho),
$$

где точка сверху обозначает дифференцирование по $z$, а $\tilde{c}$-константа интегрирования. В частности, видно, что функция $\rho^{\prime}$, получающаяся в результате преобразования Беклунда, представляет собой решение стационарного двойного уравнения синус-Гордон

$$
\ddot{\rho}+\sin \rho \cos \rho=\tilde{c} \sin \rho .
$$

Интересно отметить, что (стационарное) двойное уравнение синус-Гордон возникает в теории кристаллических дислокаций [9], [10]. В обшем случае угол $\varphi$ дается формулой

$$
\varphi=\int \Psi^{\prime}(\rho) d z-c \tilde{c} s
$$

После этого можно явно найти радиус-вектор $\mathbf{r}$. Действительно, если $\tilde{c} \neq 0$, то

$$
\mathbf{r}=\frac{1}{\tilde{c}}[\Psi(\rho) \mathbf{t}+\dot{\rho} \mathbf{n}]
$$

при этом константа интегрирования опушена. Случай $\tilde{c}=0$ можно рассмотреть, добавляя подходящую константу интегрирования и переходя к пределу $\tilde{c} \rightarrow 0$. В этом случае поток жидкости оказывается однородным. Для любой заданной пары $\{\rho, \Psi(\rho)\}$ однопараметрическое семейство волокон получается врашением одиночного волокна вокруг начала координат. Более того, скорость жидкости дается формулой

$$
\mathbf{q}=-\dot{\rho} \mathbf{t}+\Psi(\rho) \mathbf{n},
$$


а тогда из соотношения ортогональности $\mathbf{q} \cdot \mathbf{r}=0$ следует, что линии тока образуют концентрические окружности. Явно линии тока определяются формулой $z=$ const, что подтверждается соотношением

$$
\left.\frac{\partial \mathbf{r}}{\partial s}\right|_{z}=-c \mathbf{q} .
$$

В частном случае $\Psi(\rho)=\sin \rho, \mathbf{n}$-линии конвективно переносятся потоком $\mathbf{w}(4.10)$, т.е.

$$
\mathbf{w}=\mathbf{t} \operatorname{sh} \omega-\mathbf{n} \epsilon c \dot{\omega}
$$

так что

$$
\mathbf{w}=-\epsilon c \mathbf{q} .
$$

Соответственно $\mathbf{t}$ - и $\mathbf{n}$-линии можно рассматривать как два семейства волокон, которые конвективно переносятся жидкостью. Кинематика сред, армированных множественными семействами волокон, подробно исследовалась Спенсером [1]. Геометрические условия существования множественных конфигураций волокон были установлены в работе [4].

\section{Список литературы}

[1] A.J.M. Spencer. Deformations of Fibre-Reinforced Materials. Oxford Sci. Research Papers. London: Clarendon; Oxford Univ. Press, 1972.

[2] B.D. Hull, T. G. Rogers, A. J. M. Spencer. Theoretical analysis of forming flows of continuous fibre-resin systems. In: Flow and Rheology in Polymer Composites Manufacturing. Ed. S. G. Advani. Amsterdam: Elsevier, 1994. P. 203-256.

[3] A. J. M. Spencer. European J. Appl. Math. 1997. V. 8. P. 209-215.

[4] W.K. Schief, C. Rogers. The kinematics of fibre-reinforced fluids. An integrable reduction. Quart. J. Mech. Appl. Math. to appear.

[5] C. Rogers, W. K. Schief. Bäcklund and Darboux Transformations. Geometry and Modern Applications in Soliton Theory. Cambridge Texts in Applied Mathematics. Cambridge: Cambridge Univ. Press, 2002.

[6] L.P. Eisenhart. A Treatise on the Differential Geometry of Curves and Surfaces. New York: Dover, 1960.

[7] A. Sym. Soliton surfaces and their applications. In: Geometric Aspects of the Einstein Equations and Integrable Systems. Proc 6th Conf. (Scheveningen/Neth., 1984). Lect. Notes Phys. V. 239. Ed. R. Martini. Berlin: Springer, 1985. P. 154-231.

[8] A. C. Pipkin, T. G. Rogers. J. Appl. Mech. 1971. V. 38. P. 634-640.

[9] F. C. Frank, J. H. van der Merwe. Proc. Roy. Soc. London A. 1949. V. 200. P. 125-134.

[10] A. Seeger, H. Donth, A. Kochendörfer. Z. Phys. 1953. V. 134. P. 173-193. 